\title{
Silibinin Improves the Anti-arthritic Activity of Methotrexate in Zymosan-Induced Arthritis in Rats
}

\author{
Ahmed Hashem Mortada ${ }^{1}$, Saad Abdulrahman Hussain ${ }^{2, *}$ \\ ${ }^{1}$ Department of Pharmacology, College of Pharmacy, University of Kufa, Iraq \\ ${ }^{2}$ Department of Pharmacology and Toxicology, College of Pharmacy, University of Baghdad, Baghdad, Iraq \\ *Corresponding author: saad_alzaidi@yahoo.com
}

Received April 25, 2014; Revised May 12, 2014; Accepted May 12, 2014

\begin{abstract}
Because of low toxic effects of plant-derived drugs, it has become a research focus in disease prevention and treatment, especially those chronic diseases requiring long-term treatment. The present study was designed to evaluate the effects of silibinin (SIL) when administered with methotrexate (MTX) in animal model of zymosaninduced arthritis in rats. Male Wistar rats are used for induction and arthritic inflammation in the knee joint and hind paw using zymosan. The animals are treated with MTX alone $(10 \mathrm{mg} / \mathrm{kg})$, SIL $(10 \mathrm{mg} / \mathrm{kg})$ or their combination. The effect was followed through edema formation, tissue cytokines release (IL-1 $\beta$, IL-8, TNF- $\alpha$ ) and infiltration of leukocytes in synovial fluid. The results showed that SIL has weak antiarthritic activity when used alone, and improves the antiarthitic activity of MTX when both are concomitantly administered before challenge with zymosan. In conclusion, although SIL has a relatively weak anti-arthritic activity, it strongly augments the effect of MTX in animal model of zymosan-induced arthritis.
\end{abstract}

Keywords: silibinin, zymosan, arthritis, methotrexate, rats

Cite This Article: Ahmed Hashem Mortada, and Saad Abdulrahman Hussain, "Silibinin Improves the Antiarthritic Activity of Methotrexate in Zymosan-Induced Arthritis in Rats." American Journal of Pharmacological Sciences, vol. 2, no. 3 (2014): 47-51. doi: 10.12691/ajps-2-3-1.

\section{Introduction}

Rheumatoid arthritis (RA) is a systemic autoimmune disease mainly manifested as chronic, progressive and invasive arthritis [1]. The prominent clinical manifestation is recurrent multiple symmetrical small arthritis, mostly involving hands, wrists, feet and other joints; joint redness, swelling, warmth, pain and dysfunction occur in early stage, and various degrees of stiffness and deformity may occur in late stage, complicated with bone and muscle atrophy, with extremely high disability rate [2]. Regarding the pathological changes, the main pathological changes of RA include leukocytes infiltrate into articular cavity with consequent recurrent synovitis [3] and invasive pannus forms to damage cartilage, bone and surrounding tissue [4]. Early treatment of RA with non-steroidal antiinflammatory drugs (NSAIDs) can only ease pain, diminish inflammation and reduce symptoms and cannot control the activities and progress of the disease [5]. However, the use of disease modifying anti-rheumatic drugs (DMARDs) like methotrexate enables preventing joint damage [6]. Recently, new approaches are utilized in treatment of RA based targeting tumornecrosis factor antagonists (TNF- $\alpha$ ) and B-cells activity [7]. The drugs used in RA treatment are continuously updated, but adverse drug reactions are more and side effects are serious, limiting therapeutic use and clinical efficacy [8]. Silibinin (SIL) is a natural flavonoid with various biological activities [9]. Currently, silibinin preparations are advocated for the treatment of cirrhosis, chronic hepatitis and liver diseases associated with alcohol consumption and environmental toxin exposure [10]. SIL has many pharmacological effects, such as cardio protection, anti-inflammation, anti-platelet aggregation, antitumor, etc. $[11,12,13]$. SIL has very low side effects, and in human clinical trials, the recommended dose of SIL is $800 \mathrm{mg} /$ day, which corresponds to about $11.5 \mathrm{mg}$ for a $70 \mathrm{~kg}$ individual [14,15]. Because of its low toxic side effects, it has become a research focus in disease prevention and treatment, especially those chronic diseases requiring long-term treatment. Hussain etal found that SILhas anti-inflammatory effects in patients with active knee osteoarthritis [16], and Juma'a et al. reported its dose dependent effects in animal models of chronic inflammation [17]. Accordingly, the present study was designed to evaluate the effects of silibinin when administered with methotrexate in animal model of zymosan-induced arthritis in rats.

\section{Materials and Methods}

\subsection{Experimental Animals}

Male Wistar rats (200-250 g) obtained from the animal hose, College of Pharmacy, University of Baghdad were used. Animals were caged with free access to food and fresh water in a room with temperature ranging from 22 to 
$24^{\circ} \mathrm{C}$ and a $12 \mathrm{~h}$ light/dark cycle at the animal house facility, college of Pharmacy, University of Kufa. All experimental procedures were performed in accordance with the local Scientific Committee, College of Pharmacy, University of Baghdad and to the ethical guidelines of International Association for the Study of Pain [18].

\subsection{Treatments}

In the first part, animals were allocated into 4 groups (6 rats in each group). After overnight fasting, the first group received silibinin dihemisuccinate as $\beta$-Dextran complex(10 mg/kg) (TolbiacSRL, Argentina) suspended in 5\% carboxymethyl cellulose (CMC); the second group received methotrexate (10 mg/kg) (Ebwi, Austria) suspended in 5\% CMC; the third group received a mixture containing methotrexate and silibinin (10 mg/kg each); the fourth group received 5\% CMC, and served as control. All drugs and vehicle were orally administered $1 \mathrm{~h}$ prior to induction of arthritis.

\subsection{Induction of Arthritis}

During light ether anesthesia, rats received an intraarticular (i. a.) injection of zymosan (Sigma Aldrich, Tokyo, Japan) (500 $\mu \mathrm{g}$ in $25 \mu \mathrm{l}$ of sterile saline), into one knee joint. The contralateral knee was injected with the same volume of the vehicle, and used as control.

\subsection{Measurement of Knee Joint Swelling}

Knee joint swelling was evaluated by measurement of the thickness of each knee joint by a digital caliper (Digmatic Caliper, Mitsutoyo Corporation, Japan).Values of knee joint thickness were expressed as the difference $(\Delta)$ between the diameter measured before (zero time) and 6 hrs after induction of articular inflammation in millimetres (mm).

\subsection{Collection of Synovial Fluid and Leukocytesand Neutrophils Counts}

Six hours after zymosan i. a. injection, the rats were killed by injection with $100 \mathrm{mg} / \mathrm{kg}$ sodium thiopental. The synovial cavities were washed with $300 \mu$ of PBS containing EDTA $(10 \mathrm{mM})$ by the insertion of a $21 \mathrm{G}$ needle into the rat knee joints, and the synovial fluids were recovered by aspiration. Total leukocytes and neutrophils counts were performed in Neubauer chamber, under an optical microscope, after dilution in Türk fluid (2\% acetic acid). Counts are reported as numbers of cells per cavity.

\subsection{Preparation of Knee Synovial Extracts}

Knee synovial extracts were prepared as previously described by Rosengren and colleagues [19], and modified for application in rats. Rats were killed $6 \mathrm{~h}$ after zymosan i. a. injection, and the knee synovial tissue was collected. The synovial tissue was removed with a scalpel, frozen at $-40^{\circ} \mathrm{C}$ and homogenized by Teflon-head homogenizer in 1 ml HBSS containing $0.4 \%$ of triton and $0.2 \%$ of protease inhibitor cocktail (Complete Mini, Roche Applied Science, Indianapolis, IN, USA) in a rate of $50 \mu \mathrm{l}$ per $10 \mathrm{mg}$ of tissue. The homogenate was then centrifuged $(5,000 \mathrm{~g}$ for $10 \mathrm{~min}$ at $\left.4^{\circ} \mathrm{C}\right)$, the supernatant was filtered $(0.2 \mu \mathrm{l})$, stored at $-40^{\circ} \mathrm{C}$, and used later for estimation of IL-1 $\beta$, IL8 , and TNF- $\alpha$ utilizing ELISA kits according to the specifications of the manufacturer. Total protein content in the supernatant was estimated by Lowry protein assay [20].

\subsection{Zymosan-induced HindPaw Inflammation}

Twenty-four rats were allocated into 4 groups (6 rats in each) and treated as mentioned previously. To induce inflammation, rats received intraplantar injections of zymosan in $30 \mu \mathrm{l}$ of phosphate buffered saline (PBS) in the right (ipsilateral) hindpaw and $30 \mu \mathrm{l}$ of PBS in the left (contralateral) hindpaw. Hindpaw edema was measured at different time intervals (each six hrs) for $48 \mathrm{~h}$ with the digital caliper, and was expressed as percent change in dorso-ventralhindpaw thickness relative to baseline values observed before intraplantar injection.

\section{Statistical Analysis}

All the results were expressed as mean \pm S.D. Analyses were processed using Graph Pad Prism software for Windows (version 5.0, Graph Pad Software, Inc., San Diego, CA). The significance of difference among the studied groups was determined using one-way analysis of variance (ANOVA) followed by Bonferroni's post hoc test. Values with $P<0.05$ were considered significant.

\section{Results}

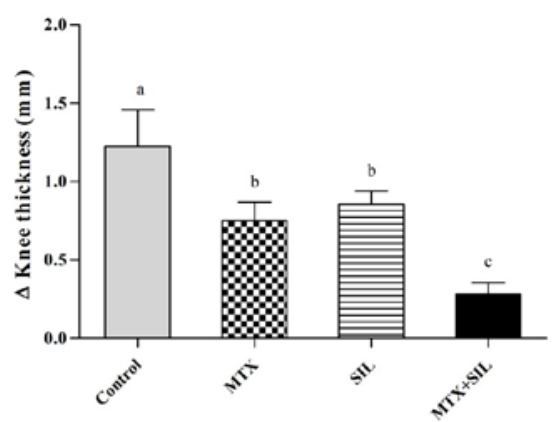

Figure 1. Effect of treatment with MTX and SIL alone or their combination on the increase in knee thickness induced by zymosan. Rats were treated $1 \mathrm{~h}$ before stimulation. Analysis was performed $6 \mathrm{~h}$ after stimulation. Results were expressed as the mean \pm S.D. from at least 6 animals per group. Statistically significant differences $(P<0.05)$ among groups are indicated by an different letters (a,b,c)

We evaluated the anti-inflammatory effects of SIL and its combination with MTX in this model of zymosaninduced articular inflammation, and observed that orally administered MTX or SIL produced comparable decrease in zymosan-induced edema formation after $6 \mathrm{~h}$, which was significantly different with that reported in control group $(P<0.05)$. Meanwhile, adjuvant use of SIL significantly improves the anti-inflammatory activity of MTX, manifested as clear reduction in zymosan-induced edema formation after $6 \mathrm{~h}$, achieving $80 \%$ of that reported in (Figure 1). In Figure 2, all treatment approached followed in the present study significantly decreased liberation of IL-1 $\beta$ in the synovial fluid compared with control, after 6 $h$ of induction with zymosan; however, adjuvant use of SIL with MTX produced maximum inhibition compared 
to the use of each one alone. Figure 3 shows that both MTX and SIL produced comparable inhibitory effect on the release of IL- 8 in the inflamed synovium $6 \mathrm{~h}$ after induction with zymosan, while concomitant oral administration of both agents produced maximum inhibition (64\%) of IL-8 release, which was significantly different compared with other approaches. Similarly, adjuvant use of SIL with MTX results in a highly significant reduction in TNF- $\alpha$ release in synovial fluid after induction with zymosan compared with control and both MTX and SIL when each one administered alone (Figure 4).The induction of inflammation wit i. a. injection of zymosan produced an important increase in total leukocyte numbers in the articular space within $6 \mathrm{~h}$ (Figure 5). Such increase seems to be due to the influx of neutrophils (Figure 6). Interestingly, pre-treatment with a combination of MTX and SIL significantly inhibited the increase in totalleukocyte and neutrophil numbers in the synovial fluid, which was greater than that produced when MTX or SIL administered alone (Figure 5 and Figure 6). In zymosan-induced edema in rats' hind paw, Figure 7 clearly shows that co-administration of MTX and SLI results in greater attenuation of edema formation after induction with zymosan during $48 \mathrm{~h}$ post-challenge. This effect seems significantly higher than control and animal groups treated with either MTX different or SIL alone at different time intervals (12, 24, 36, and 48 h); meanwhile, the oral dose of SLI alone did not show significant reduction in edema formation during all time intervals compared with control.

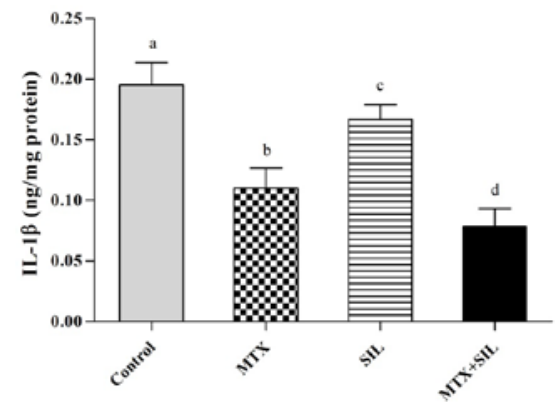

Figure 2. Effect of treatment with MTX and SIL alone or their combination on IL-1 $\beta$ generation induced by zymosan in rats, determined by ELISA in tissue extractsof knee joints recovered $6 \mathrm{~h}$ after zymosan stimulation. Results are expressed as the mean \pm S.D. from at least6 animals per group. Statistically significant differences $(P<0.05)$ among groups were indicated by different letters (a,b,c,d)

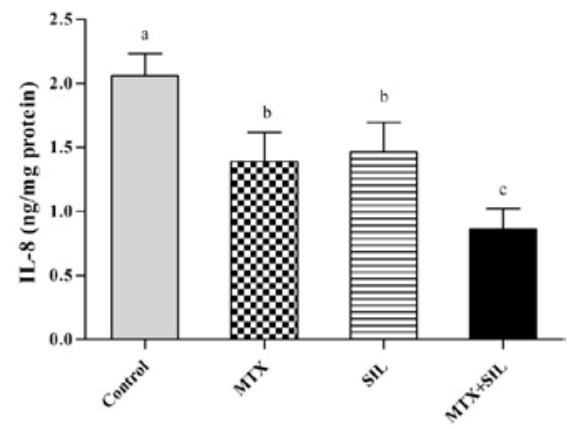

Figure 3. Effect of treatment with MTX and SIL alone or their combination on IL-8 generation induced by zymosan in rats, determined by ELISA in tissue extracts of knee joints recovered $6 \mathrm{~h}$ after zymosan stimulation. Results are expressed as the mean \pm S.D. from at least 6 animals per group. Statistically significant differences $(P<0.05)$ among groups were indicated by different letters (a,b,c)

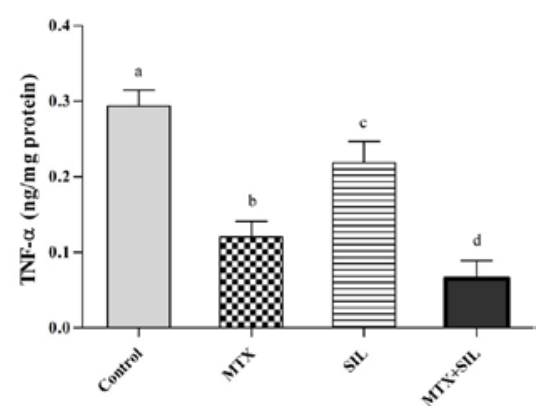

Figure 4. Effect of treatment with MTX and SIL alone or their combination on TNF- $\alpha$ generation induced by zymosan in rats, determined by ELISA in tissue extracts of knee joints recovered $6 \mathrm{~h}$ after zymosan stimulation. Results are expressed as the mean \pm S.D. from at least 6 animals per group. Statistically significant differences $(P<0.05)$ among groups were indicated by different letters (a,b,c,d)

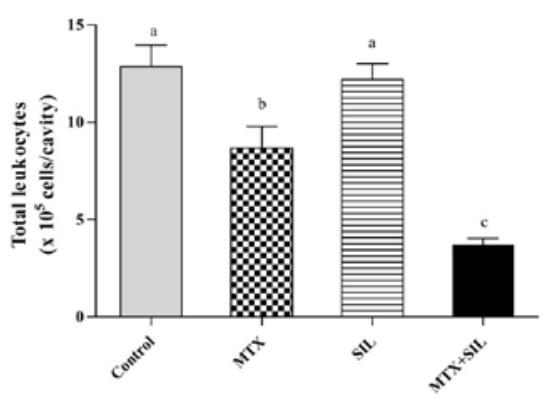

Figure 5. Effect of treatment with MTX and SIL alone or their combination on total leukocytes accumulation into rats' synovial space6 $\mathrm{h}$ after zymosan i. a. injection. Rats were treated $1 \mathrm{~h}$ beforestimulation. Results were expressed as the mean \pm S.D. from at least6 animals per group. Statistically significant differences $(P<0.05)$ among groups were indicated by an different letters (a,b,c)

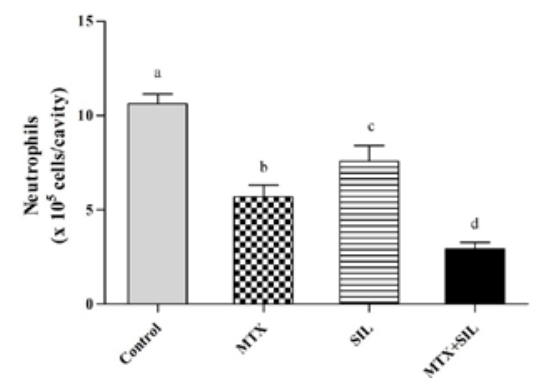

Figure 6. Effect of treatment with MTX and SIL alone or their combination on neutrophils accumulation into rats' synovial space $6 \mathrm{~h}$ after zymosan i. a. injection. Rats were treated $1 \mathrm{~h}$ before stimulation. Results were expressed as the mean \pm S.D. from at least 6 animals per group. Statistically significant differences $(P<0.05)$ among groups were indicated by an different letters (a,b,c,d)

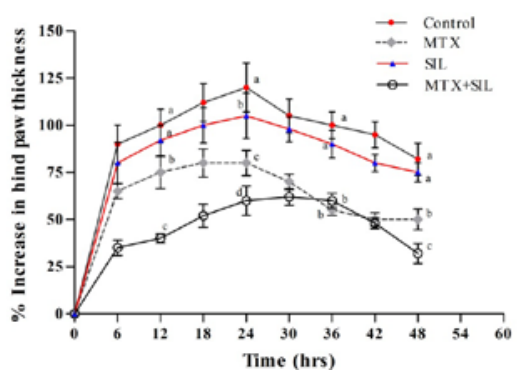

Figure 7. Time-course of zymosan-induced increase in pawthickness in rats treated with MTX, SIL or their combination. Analysis was performed from $6 \mathrm{~h}$ to $48 \mathrm{~h}$ after stimulation. Results are expressed as the mean \pm S.D.from at least 6 animals per group. Statistically significant differences $(P<0.05)$ among groups are indicated by different letters (a,b,c,d) 


\section{Discussion}

Zymosan, a cell wall component of Saccharomyces cerevisiae, is a crude extract that contains carbohydrate components, such as $\beta$-glucan. These components are responsible for innate immune induction via production of prostanoids, activation of the complement system and direct activation of immune cells via the $\beta$-glucan receptor Dectin-1 [21,22]. In the present study, the reported changes in the knee joints and hind paw are identical with the picture of arthritis reported in genetically modified mice, which represent the typical model of zymosaninduced arthritis [23]. The results clearly showed that MTX efficiently suppressed the inflammatory response when dosed prophylactically, revealed in both edema formation and cytokines production. One of the proposed targets of MTX activity is suppression of T cells [24]. As the pathology of SKG mice is dependent on auto-reactive $\mathrm{T}$ cells presumably by genetic alteration [15], it is reasonable to hypothesize that the MTX mechanism of action in this model is directly mediated by its effect on the irregular $\mathrm{T}$ cells circulating in SKG mice. Many experimental animals' studies of natural products therapies have been conducted because of the wide range of adverse effects associated with the long-term use of different types of anti-inflammatory drugs reported during the clinical practice [25]. Although animal models have many limitations, they have contributed to the basic understanding of inflammatory joint diseases and development of drugs with effective anti-inflammatory and anti-arthritic activities [17,26]. In the present study, although SILshows relatively weak anti-arthritic activity, when administered prophylactically as single oral dose, it significantly augment the effect of MTX, when administered concomitantly, revealed as attenuation of edema formation in the knee and hind paw, cytokine release in synovial tissue, and leukocyte migration in the knee joint induced by zymosan. Silymarin, an extract composed of $>80 \%$ SIL, at doses 6.25, 12.5 and $25 \mathrm{mg} / \mathrm{kg}$ when administered orally, reported to produce antiarthritic activity in Mycobacterial adjuvant-induced arthritis model in rats in a dose-dependent manner [27]. This effect may be attributed to the inhibition of many enzymes and mediators involved in inflammation. The clinical application of this concept in the treatment of osteoarthritis resulted in a promising effects marking silymarin as a good candidate for the treatment of inflammatory joint diseases[16]. During the course of zymosan-induced arthritic lesion, the released cytokines TNF- $\alpha$ and IL- $1 \beta$ are agonists of NF- $\kappa B$ and target genes to induce transcription. Thus, NF- $\kappa \mathrm{B}$ activation leads to increased TNF- $\alpha$ and IL-1 $\beta$ expression and are further acting on NF- $\kappa B$, to make its activity more enhanced. SIL can regulate $\mathrm{NF}-\kappa \mathrm{B}$ activity [28], and through the regulation of $\mathrm{NF}-\kappa \mathrm{B}$ and inhibition of inflammatory cytokine expression, achieve the effects of preventing inflammation and reducing inflammatory reactions. Moreover, NF- $\kappa \mathrm{B}$ contributes to the production of IL-1, IL-6, TNF- $\alpha$, lymphotoxin, granulocyte-macrophage colony-stimulating factor (GM-CSF) and interferon gamma (IFN- $\gamma)$ [29]. Furthermore, some of these cytokines, e.g., IL-1 and TNF- $\alpha$, activate NF- $\kappa \mathrm{B}$ themselves, thus creating positive feedback loop and interruption of this loop by SIL might be responsible for its chronic anti-inflammatory activity [30]. Silibinin also has an important immunomodulatory function through inhibition of dendritic cell maturation under both in vitro and in vivo conditions [31] and also inhibit TNF- $\alpha$ secretion by human T-cells tested freshly in vitro [28]. Thus, immunosuppression is considered as a common feature between disease modifying antirheumatic drugs including MTX and silymarin, and this could explain the relative similarity in action between MTX and SIL especially at a dose $10 \mathrm{mg} / \mathrm{kg}$ in zymosan-induced arthritis model in rats. Several human arthropathies, including RA are characterized by significant joint edema, movementinduced joint hyperalgesia and the infiltration of inflammatory cells [32]. The role of neutrophils in the pathogenesis of arthritic lesions has long been recognized [33].The experimental model of zymosan-induced arthritis is associated with neutrophil recruitment, lymphocyte proliferation, and synovial hypertrophy [34]. In the present study, SILsignificantly inhibited leukocyte migration to the articular cavity in rats, and augment the effect of MTX in this regard. Experimental arthritis promoted the accumulation of inflammatory cells in the synovial fluid. Zymosan, which is a ligand for toll-like receptor 2 and activator of the alternative complement pathway, triggers local activation of the innate immune system, causing inflammation in the injected joint [35]. At early time points after zymosan injection, edema formation is accompanied by neutrophil infiltration and the release of inflammatory mediators in the synovial tissue and fluid in the inflamed joints. In the present study, the effect of zymosan injection was observed as an intense inflammatory reaction, characterized by increased leukocyte migration in the articular cavity. Furthermore, we found that oral treatment with a combination of MTX and SIL as a single dose significantly decreased leukocyte (specifically neutrophils) migration in the synovial fluid, which may attenuate edema formation and cytokine release compared with the control group. In the present study, the inflammatory response after an intraplantar injection of zymosan into the right hind paw showed a time-dependent response, with maximal swelling at 18-24 $\mathrm{h}$ that started to gradually decline toward baseline. Zymosan-induced paw inflammation in miceinduces peripheral edema, COX-2 expression, primary hyperalgesia, and central sensitization [36]. In the present study, MTX, SIL or their combination, reduced paw edema in mice with a maximal effect at $18 \mathrm{~h}$ (Figure 7). This anti-edematous effect suggests that the inhibitory action of the combination was significantly greater than the effects of both agents, when each one administered alone, and may be attributable to the suppression of the release of many mediators responsible for inflammation, including PGE2 and COX-2 [37], in addition to those reported in the present study. In conclusion, although silibinin has a relatively weak anti-arthritic activity, it strongly augments the effect of methotrexate in animal model of zymosan-induced arthritis.

\section{Acknowledgement}

The data was abstracted from $\mathrm{PhD}$ thesis submitted by Ahmed H. Mortada to the Department of Pharmacology and Toxicology, College of Pharmacy, University of 
Baghdad. The authors thank Bushra H. Marouf for technical assistance, and the University of Baghdad for supporting the project.

\section{References}

[1] Firestein GS. Evolving concepts of rheumatoid arthritis. Nature, 423: 356-361. 2003.

[2] Hootman J, Helmick C. Projections of US prevalence of arthritis and associated activity limitations. Arthritis Rheum., 54: 226-229. 2006.

[3] Cooles FA, Isaacs JD. Pathophysiology of rheumatoid arthritis. Curr. Opin. Rheumatol., 23: 233-240. 2011.

[4] Szekanecz Z, Koch AE. Update on synovitis. Curr. Rheumatol. Rep., 3: 53-63. 2001.

[5] Smolen JS, Landewe R, Breedveld FC, Dougados M, et al. EULAR recommendations for the management of rheumatoid arthritis with synthetic and biological disease-modifying antirheumatic drugs. Annals. Rheum. Dis., 69: 964-975. 2010.

[6] Choy EH, Panayi GS. Cytokine pathways and joint inflammation in rheumatoid arthritis. N. Engl. J. Med., 344: 907-916. 2001.

[7] Gibbons LJ, Hyrich KL. Biologic therapy for rheumatoid arthritis: clinical efficacy and predictors of response. Bio. Drugs Clin. Immunother. Biopharm. Gene Ther., 23: 111-124. 2009.

[8] Mease PJ, Cohen S,Gaylis NB, Chubick A, et al. Efficacy and safety of retreatment in patients with rheumatoid arthritis with previous inadequate response to tumor necrosis factor inhibitors: results from the SUNRISE trial. J. Rheumatol., 37: 917-927. 2010.

[9] Kren V, Walterov D. Silybin and Silymarin- New effects and applications. Biomed. Papers, 149 (1): 29-41. 2005.

[10] Fraschini F, Dermartini G, Esposti D. Pharmacology of silymarin. Clin. Drug Invest., 22: 51-65. 2002.

[11] Vereckei AS, Besch HR, Zipes DP. Combined amiodarone and silymarin treatment, but not amiodarone alone, prevents sustained atrial flutter in dogs. J. Cardiovasc. Electrophysiol., 14: 861-867. 2003.

[12] Singh RP, Mallikarjuna GU, Sharma G, Dhanalakshmi S, et al. Oral silibinin inhibits lung tumor growth in athymic nude mice and forms a novel chemocombination with doxorubicin targeting nuclear factor $\kappa \mathrm{B}$-mediated inducible chemoresistance. Clin. Cancer Res., 10: 8641-8647. 2014.

[13] Kang JS, Park SK, Yang KH, Kim HM. Silymarin inhibits TNF- $\alpha-$ induced expression of adhesion molecules in human umbilical vein endothelial cells. FEBS Lett., 550: 89-93. 2002.

[14] Jacobs BP, Dennehy C, Ramirez G, Sapp J, Lawrence VA.Milk thistle for the treatment of liver disease: A systematic review and meta-analysis.Am. J. Med., 113: 506-515. 2002.

[15] Invernizzi R, Bernuzzi S, Ciani D, Ascari E. Silymarin during maintenance therapy of acute promyelocytic leukemia. Haematologica, 78: 340-341. 1993.

[16] Hussain SA, Jassim NA, Numan IT, Al-Khalifa II, Abdullah TA. Anti-inflammatory activity of silymarin in patients with knee osteoarthritis: A comparative study with piroxicam and meloxicam. Saudi Med. J., 30 (1): 179-184. 2009.

[17] Juma'a KM, Ahmed ZA, Numan IT, Hussain SA.Dose-dependent anti-inflammatory effect of silymarin in experimental animal model of chronic inflammation. Afr. J. Pharmacy Pharmacol., 3 (5): 242-247. 2009.
[18] Zimmermann M. Ethical guidelines for investigation of experimental pain in conscious animals. Pain, 16: 109. 1993.

[19] Rosengren S, Firestein GS, Boyle DL. Measurement of inflammatory biomarkers in synovial tissue extracts by enzymelinked immunosorbent assay. Clin. Diagn. Lab. Immunol., 10: 1002-1010. 2003.

[20] Lowry OH, Rosebrough NJ, Farr AL, Randall RJ.Protein measurement with Folin phenol reagent.J. Biol. Chem., 193: 265275. 1951.

[21] Kobayashi K, Suda T, Nanya K, Sakaguchi N, et al. Cytokine production profile of splenocytes derived from zymosan-treated SKG mice developing arthritis. Inflamm. Res., 55: 335-341. 2006.

[22] Yuhki KI, Ushikubi F, Naraba H, Ueno A, et al. Prostaglandin I2 plays a key role in Zymosan A-induced mouse pleurisy. $J$. Pharmacol. Exp. Ther., 325: 601-609. 2008.

[23] Kannan K, Ortmann RA, Kimpel D. Animal models of rheumatoid arthritis and their relevance to human disease. Pathophysiology, 12: 167-181. 2005.

[24] Nagate T, Tamura T, Sato F, Kuroda J, et al. Tranilast suppresses the disease development of the adjuvant- and streptococcal cell wall-induced arthritis in rats. J. Pharmacol. Sci., 105: 48-56. 2007.

[25] Venkatesha SH, Rajaiah R, Berman BM, Moudgil KD. Immunomodulation of autoimmune arthritis by herbal cam. Evid. Based Complement. Alternat. Med., 11: 797-803. 2011.

[26] Jung HW, Jung JK, Son KH, Lee DH, et al. Inhibitory effects of the root extract of Dipsacusasperoides on collagen-induced arthritis in mice. J. Ethnopharmacol., 139: 98-103. 2012.

[27] Gupta OP, Sing S. Anti-inflammatory and antiarthritic activities of silymarin acting through inhibition of 5-lipoxygenase. Phytomedicine, 7 (1): 21-24. 2000.

[28] Polyak SJ, Morishima C. Inhibition of T-cell inflammatory cytokines, hepatocyte NF- $\mathrm{kB}$ signaling and $\mathrm{HCV}$ infection by standardized silymarin. Gastroenterology, 132: 1925-1936. 2007.

[29] Barnes PJ, Karin M. NF- $\mathrm{kB}$ a pivotal transcription factor in chronic inflammatory diseases. N. Eng. J. Med., 336: 1066-1071. 1997.

[30] Monna SK, Mukhopadhya A, Van NT. Silymarin suppresses TNFinduced activation of NF-KB, c-Jun N-terminal kinase and apoptosis. J. Immunol., 163 (12): 6800-6809. 1999.

[31] Lee JS, Kim SG. Silibinin polarize Th1/Th2 immune responses through the inhibition of immunostimulatory function of dendritic cells. J. Cell Physiol., 210: 385-397. 2007.

[32] Kraan MC, Haringman JJ, Ahern MJ, Breedveld FC, et al. Quantification of the cell infiltrate in synovial tissue by digital image analysis. Rheumatology, 39: 43-49. 2000.

[33] Jonsson H, Allen P, Peng SL. Inflammatory arthritis requires Foxo3a to prevent Fas ligand-induced neutrophil apoptosis. Nat. Med., 11: 666-671. 2005.

[34] Asquith DL, Miller AM, McInnes IB, Liew FY.Animal models of rheumatoid arthritis.Eur. J. Immunol., 39: 2040-2044. 2009.

[35] Frasnelli ME, Tarussio D, Chobaz-Peclat V, Busso N, So A. TLR2 modulates inflammation in zymosan-induced arthritis in mice. Arthritis Res. Ther., 7: 370-379. 2005.

[36] Jain NK, Ishikawa TO, Spigelman I, Herschman HR. COX-2 expression and function in the hyperalgesic response to paw inflammation in mice. Prostaglandins Leukot. Essent. Fatty Acids, 79: 183-190. 2008.

[37] Setty AR, Sigal LH. Herbal medications commonly used in the practice of rheumatology: mechanisms of action, efficacy, and side effects. Semin. Arthritis Rheum., 34: 773-784. 2005. 